\title{
Dermatophytes and other associated fungi in patients attending to some hospitals in Egypt
}

\author{
Al Shimaa M. Abd Elmegeed ${ }^{1}$, S.A. Ouf ${ }^{2}$, Tarek A.A. Moussa ${ }^{2}$, S.M.R. Eltahlawi ${ }^{3}$ \\ ${ }^{1}$ Medical biology Department, Faculty of medicine, Gizan University, Gizan, Saudi Arabia. \\ ${ }^{2}$ Botany Department, Faculty of Science, Cairo University, Giza, Egypt. \\ ${ }^{3}$ Dermatology Department, Faculty of Medicine, Cairo University, Giza, Egypt.
}

Submitted: July 22, 2014; Approved: November 28, 2014.

\begin{abstract}
Dermatophytes are keratinophilic fungi that infect keratinized tissues causing diseases known as dermatophytoses. Dermatophytes are classified in three genera, Epidermophyton, Microsporum, and Trichophyton. This investigation was performed to study the prevalence of dermatomycosis among 640 patients being evaluated at the dermatology clinics at Kasr elainy, El-Husein and Said Galal hospitals in Cairo and Giza between January 2005 and December 2006. The patients were checked for various diseases. Tinea capitis was the most common clinical disease followed by tinea pedis and tinea corporis. Tinea cruris and tinea unguium were the least in occurrence. Tinea versicolor also was detected. The most susceptible persons were children below 10 years followed by those aged 31-40 years. Unicellular yeast was the most common etiological agent and $T$. tonsurans was the second most frequent causative agent followed by M. canis.
\end{abstract}

Key words: dermatophytosis, dermatophytes, fungi, keratinophilic, prevalence, dermatology.

\section{Introduction}

Dermatophytes are a group of closely related keratinophilic fungi that infect keratinized tissues such as hair, nails and skin. The disease caused by dermatophytes is known as dermatophytosis which constitutes an important public health problem, not only in underdeveloped countries but also in elderly and immuno-compromised patients worldwide (Walsh and Groll, 1999; Ghannoum et al., 2003; Carrillo-Munoz et al., 2008).

The etiologic agents of the dermatophytosis can be categorized into one of three genera: Epidermophyton, Microsporum and Trichophyton (Ghannoum and Isham, 2009). They possess keratinophilic and keratinolytic properties (Simpanya, 2000).

Traditionally, infections caused by dermatophyte (ring-worm) have been named by appending the latin name of the affected body part after the word "tinea" (Andrews and Burns, 2008).

Tinea capitis (ringworm of the scalp) is the most common fungal infection in children. More than $90 \%$ of the in- fections are caused by Trichophyton tonsurans, and fewer than 5\% are caused by Microsporum species (Andrews and Burns, 2008). Tinea barbae, an infection of the bearded area in the adult males. Lesions are severe pustular eruption, deep inflammatory plaques and non-inflammatory superficial patches (Baran et al., 2004). It more commonly caused by $T$. verrucosum, $T$. mentagrophytes var. granulosum (Kwon-Chung and Bennett, 1992; Baran et al., 2004). Tinea corporis usually involving the trunk, limbs, and occasionally the face (Weitzman and Summerbell, 1995). The infection commonly appears as annular, scaly patches or plaque with raised, scaling border and central clearing. $T$. rubrum is the most common cause worldwide (Andrews and Burns, 2008). In areas where tinea capitis is endemic, tinea corporis is more commonly caused by $T$. tonsurans, Other causative dermatophytes include $M$. audouinii, $T$. mentagrophytes, T. verrucosum, and E. floccosum (Denk, 2007). Tinea cruris is infection of the groin, perianal, and perineal areas and usually occurring predominantly in adolescent, young adult men and in post-pubertal females. $T$. 
rubrum is the most common causative agent followed by $E$. floccosum (Chakrabarti et al., 1992). Tinea Versicolor is a superficial fungal infection of the skin produced by Malassezia (lipophilic dimorphic fungi). It presents as small or medium sized circular or oval, erythematous, hyper- or hypo-pigmented macules. The most frequently affected areas are those supplied by the sebaceous glands, mainly the upper third of the trunk, especially the shoulder, proximal upper extremities, the neck, and less frequently, the face (Fernández-Vozmediano and Armario-Hita, 2006). Tinea imbricate, the chronic infection which is a specialized manifestation of tinea corporis. $T$. concentricum is the only etiologic agent (Mousavi et al., 2009). Tinea manuum, appears as diffuse dry scaling lesions, with accentuation of the flexural creases of the palms of the hands.T. rubrum is the commonest infecting agent (Degreef, 2008). Tinea pedis, usually originates in the interdigital clefts, sometimes spreading to the soles, dorsum and occasionally the ankles, leg and ultimately to the toenails, resulting in tinea unguium (Baxter and RushMunro, 1980). The presence of diabetes mellitus is a risk factor for tinea pedis (Porche, 2006). Seebacher et al. (2008) reported that the fungal biota of tinea pedis consisted in three different fungal species, T. rubrum $(72.9 \%)$, T. mentagrophytes $(16.6 \%)$ and E. floccosum. Tinea unguium (Onychomycosis), fungal infection of the nail caused mainly by $T$. rubrum and T. mentagrophytes var. interdigitale (Svejgaard and Nilsson, 2004; Dolenc-Voljc, 2005; Mugge et al., 2006).

The aim of this investigation was to study the occurrence, distribution and prevalence of dermatophytes causing human dermatomycosis in the selected group of patients.

\section{Materials and Methods}

\section{Population study and clinical assessment}

640 patients from Cairo and Giza city suffered from different types of skin mycosis were evaluated at the dermatology clinics at Kasr elainy, El-Husein and Said Galal hospitals from January 2005 to December 2006 of which 310 were males and 330 females. The population surveyed was classified according to age which ranged from 4 months to 70 years into seven groups spanning 10 years each. The assessment of the participants consisted of an interview, clinical examination and collection of specimens for microbiological studies. All patients completed a questionnaire that contained demographic data, patient \& family history and specific data related to risk factor for dermatophytosis and candidiasis such as age, gender, physical activities, occupation, predisposing diseases and contact with animals and clinical diagnosis.

\section{Collection and transport of specimens}

The suspected ringworm lesions were cleaned with $70 \%$ ethyl alcohol using sterile cotton. Sample materials were transported in dry, strong black paper folded in the manner of a herbarium packet and transferred to the laboratory as soon as possible for direct microscopic examination and culturing (Weitzman and Summerbell, 1995; Kane and Summerbell, 1997).

For tinea corporis and tinea cruris, the best collection is made by scraping of epidermal scales using a sterile scalpel blade from near the advancing edges of ringworm (Weitzman and Summerbell, 1995). In tinea capitis, hair are best sampled by plucking so that the root is included. The basal root portion of the hair is best for direct microscopy and culture. In 'black dot' tinea capitis, a scalpel may be used. In tinea unguium, the common distalsubungual type is traditionally sampled by scraping the debris from beneath the distal end of the nail with a scalpel near the nail bed. Superficial white onychomycosis is sampled by scraping material from the white spots on the surface of the nail (Weitzman and Summerbell, 1995). In tinea pedis, a sample from the fourth toe clefts of both feet is taken (Auger et al., 1993). In cases of pityriasis versicolor, when very little scaling is present, it is possible to take a sample by pressing a strip of sticky tape (Sellotape) onto the lesion for the direct examination.

\section{Microscopic examination of specimens}

Was performed following treatment with an aqueous solution of $20 \%$ potasium hydroxide $(\mathrm{KOH})$ mixed with $5 \%$ glycerol and heated for $1 \mathrm{hr}$ at $50{ }^{\circ} \mathrm{C}$ (Rebell and Taplin, 1970).

\section{Culturing of specimens}

All samples were cultured on SDA (CM41; Oxoid, Basingstoke, United Kingdom) supplemented by chloramphenicol (RS78; Oxoid, Basingstoke, United Kingdom) and cycloheximide (RS222; Oxoid, Basingstoke, United Kingdom) (Rebell and Taplin, 1970). It is critical to use a cycloheximide-free medium when non-dermatophytic fungi or yeasts other than Candida albicans are suspected to be etiologic agents. The plates were inoculated and incubated at $25^{\circ} \mathrm{C}$ or $30^{\circ} \mathrm{C}$ for up to 4 weeks.

\section{Examination and identification of fungus isolates}

Identification of characters included macroscopic and microscopic examination using references from Summerbell (2003) and Zagnoli et al. (2005). 


\section{Results}

\section{Clinical types according to age}

Table 1 shows a survey of 640 patients from three hospitals suffering from skin mycosis induced by dermatophytes and other fungi. The data has been classified according to age group. The data indicate that tinea capities was the most common occurrence representing $28.6 \%$ of the cases, followed by tinea pedis $(21.1 \%)$ and tinea corporis $(14.8 \%)$. Tinea cruris and tinea unguium were the least in occurrence among patients at 6.9 and $7.7 \%$. Tinea versicolor was detected in 74 cases representing 11.6\%. The skin diseases due to Candida spp. represented $9.4 \%$ of skin mycosis. All clinical types of tinea, except tinea capitis and tinea versicolor were more common in males than in females. The most susceptible age group was that of children below 10 years of age. (286 cases accounting for $44.7 \%$ ) followed by those aged between 31-40 years ( 96 cases accounting for $15 \%)$. Skin mycosis was rarely reported in older persons of age groups 61-70 and 51-60 years.

Table 2 shows the classification of tinea corporis according to the infection site and age group. More than 50\% of tinea corporis cases were estimated in children between 4 months and 10 years and patients were significantly more likely to be male than female. Face and neck were frequently the target of infection followed by trunk and shoulders. Cases of axillae and legs were less common.

\section{Clinical types according to etiological agents}

Diagnosis of infection was confirmed by microscopic examination in all cases and the causative agent was iso- lated and cultured on suitable medium for identification. The unicellular yeast was the most commonly identified etiological agent in all clinical types of tinea and candidiasis (181 cases accounting for $28.3 \%$ of the total cases) (Table 3).

More than one hundred of yeast cases were identified in both candidiasis and tinea pedis. T. tonsurans was ranked the second most frequent causative agent being identified in 119 cases followed by M. canis, 85 cases T. tonsurans was responsible for 83 cases of tinea capitis, 34 cases of tinea corporis and two cases of tinea cruris, while $M$. canis was responsible for 61 cases of tinea capitis and 24 cases of tinea corporis. T. mentagrophytes (49 cases) and T. rubrum (39 cases), both were recovered from tinea cruris and tinea pedis. M. gypseum and Malassezia fufur were only detected in tinea capitis ( 14 cases) and tinea versicolor (53 cases), respectively. Filamentous non-dermatophytes including Aspergillus spp., Scopulariopsis spp. and Fusarium spp. were rare as causative agents and all recovered in 29 cases representing $4.5 \%$ of the total cases.

\section{Discussion}

Dermatophytes (Trichophyton, Microsporum and Epidermophyton) invade the keratinized tissues and cause dermatophytosis (Weitzman and Summerbell, 1995). The prevalence of dermatophytosis has increased worldwide in recent years, especially in immunocompromised patients (Arrese et al., 2005; Borman et al., 2007).

In the present research, population study and clinical assessment of different types of skin mycosis and their etio-

Table 1 - Survey of patients suffering from skin mycosis due to dermatophytes and other fungi in three hospitals in Egypt according to age group.

\begin{tabular}{|c|c|c|c|c|c|c|c|c|c|c|}
\hline \multicolumn{2}{|c|}{ Clinical typesof tinea } & \multicolumn{7}{|c|}{ Age groups (yr) } & \multirow{2}{*}{$\begin{array}{l}\text { Total male/ } \\
\text { total female }\end{array}$} & \multirow[t]{2}{*}{ Total } \\
\hline & & $0-10$ & $11-20$ & $21-30$ & $31-40$ & $41-50$ & $51-60$ & $61-70$ & & \\
\hline \multirow[t]{2}{*}{ Tinea capitis } & Male & 98 & 22 & - & - & - & - & - & 120 & 183 \\
\hline & Female & 63 & - & - & - & - & - & - & 63 & \\
\hline \multirow[t]{2}{*}{ Tinea corporis } & Male & 28 & 8 & 2 & - & 6 & - & - & 44 & 95 \\
\hline & Female & 20 & 3 & 4 & 14 & 6 & 4 & - & 51 & \\
\hline \multirow[t]{2}{*}{ Tinea cruris } & Male & 4 & 2 & 8 & 2 & 2 & 2 & 1 & 21 & 44 \\
\hline & Female & 3 & 2 & 5 & 5 & 8 & - & - & 23 & \\
\hline \multirow[t]{2}{*}{ Tinea pedis } & Male & 4 & 5 & 8 & 10 & 8 & 5 & 5 & 45 & 135 \\
\hline & Female & - & - & 12 & 29 & 32 & 16 & 1 & 90 & \\
\hline \multirow[t]{2}{*}{ Tinea unguium } & Male & - & - & 2 & 6 & 4 & 1 & - & 13 & 49 \\
\hline & Female & 1 & 1 & 2 & 14 & 16 & 2 & - & 36 & \\
\hline \multirow[t]{2}{*}{ Tinea versicolor } & Male & - & 20 & 16 & 5 & - & - & - & 41 & 74 \\
\hline & Female & 6 & 9 & 7 & 11 & - & - & - & 33 & \\
\hline \multirow[t]{2}{*}{ Candidiosis } & Male & 26 & - & - & - & - & - & - & 26 & 60 \\
\hline & Female & 33 & 1 & - & - & - & - & - & 34 & \\
\hline Total & & 286 & 73 & 66 & 96 & 82 & 30 & 7 & $310 / 330$ & 640 \\
\hline
\end{tabular}


Table 2 - Classification of tinea corporis according to infection site and age group.

\begin{tabular}{|c|c|c|c|c|c|c|c|c|c|c|c|c|}
\hline \multirow[t]{3}{*}{ Age groups (yr) } & \multicolumn{10}{|c|}{ Number of males and females according to sites of infection } & \multirow{3}{*}{$\begin{array}{l}\text { Total M / } \\
\text { total F }\end{array}$} & \multirow[t]{3}{*}{ Total } \\
\hline & \multicolumn{2}{|c|}{ Face, neck } & \multicolumn{2}{|c|}{ Trunk } & \multicolumn{2}{|c|}{ Shoulders } & \multicolumn{2}{|c|}{ Axillae } & \multicolumn{2}{|c|}{ Legs } & & \\
\hline & $\mathrm{M}^{*}$ & $\mathrm{~F}^{* *}$ & M & $\mathrm{F}$ & M & $\mathrm{F}$ & M & $\mathrm{F}$ & M & $\mathrm{F}$ & & \\
\hline $0-10$ & 20 & 10 & 4 & 6 & 2 & 4 & 1 & - & 1 & - & $28 / 20$ & 48 \\
\hline $11-20$ & 6 & 2 & 1 & - & 1 & - & - & - & - & 1 & $8 / 3$ & 11 \\
\hline $21-30$ & - & 2 & 1 & - & - & 2 & - & - & 1 & - & $2 / 4$ & 6 \\
\hline $31-40$ & - & 7 & - & 2 & - & 4 & - & - & - & 1 & $-/ 14$ & 14 \\
\hline $41-50$ & - & 3 & 2 & 1 & 3 & 1 & 1 & 1 & - & - & $6 / 6$ & 12 \\
\hline $51-60$ & - & - & - & 1 & - & 1 & - & 2 & - & - & $-/ 4$ & 4 \\
\hline $61-70$ & - & - & - & - & - & - & - & - & - & - & $-/-$ & - \\
\hline \multirow[t]{2}{*}{ Total } & 26 & 24 & 8 & 10 & 6 & 12 & 2 & 3 & 2 & 2 & $44 / 51$ & 95 \\
\hline & \multicolumn{2}{|c|}{50} & \multicolumn{2}{|c|}{18} & \multicolumn{2}{|c|}{18} & \multicolumn{2}{|c|}{5} & \multicolumn{2}{|c|}{4} & & \\
\hline
\end{tabular}

*Male, **Female.

logical agents was done in 640 patients aged between 4 months to 70 years in three hospitals at Cairo and Giza city. Tinea capitis followed by tinea pedis and tinea corporis were the most common types of tinea infection. During the last few decades, a substantial increase in the prevalence of mycotic scalp infection and a remarkable change in the pattern of the causative dermatophytes among different developed countries has been observed (Ginter-Hanselmayer et al., 2007; Raccurt et al., 2009). The incidence of tinea capitis varies according to the climate, temperature, relative humidity, economic status, and precipitation of different geographic regions, as well as, the natural reservoir of infection (Moraes et al., 2006; Ginter-Hanselmayer et al., 2007; Ngwogu and Otokunefor, 2007; Samarai, 2007).

The most susceptible persons to tinea capitis were children below 10 years because of the lack of protective fatty acids in their scalp. This infection was rarely reported in persons above fifty years of age. Earlier, several authors have corroborated this finding. Some factors implicated in infection include poor personal hygiene, crowded living conditions, and low socioeconomic status. Rebollo et al. (2008) reported that tinea capitis is mostly exclusive to children and rarely occurs after puberty, probably due to changes in the $\mathrm{pH}$ of the scalp and an increase in fatty acids serving a protective role. Consequently, most cases occurring in adults involve women with hormonal disorders resulting in carryover of tinea capitis from childhood or in patients with severe immunodepression due to leukemia, lymphoma, or treatment with immunosuppressant drugs.

Species identification of tinea capitis showed that $T$. tonsurans (83 cases) was the most common dermatophytes followed by $M$. canis (61 cases) and M. gypseum (14 cases). Several investigators reported that tinea capitis is caused by a number of Trichophyton and Microsporum species. The anthropophilic Microsporum species cause a contagious disease, endemic in many countries. The zoophilic Trichophyton and Microsporum species are seldom responsible for more than minor outbreaks of human infections. M. canis, T. mentagrophytes, $T$. verrucosum, T. tonsurans, $T$. violaceum, and $M$. audouinii species are causal agents of tinea capitis (Arenas et al., 2006; Ilkit et al., 2007). However, Rebollo et al. (2008) reported that in developed countries, $T$. tonsurans is the most common causative agent of tinea capitis, whereas in developing countries such as Mexico, the most common agent is $M$. canis followed by $T$. tonsurans.

In the Middle East, T. violaceum is responsible for most cases of tinea capitis, accounting for $83 \%$ of cases in the West Bank of Palestine and for 39\% in Iraq (Al-Duboon et al., 1999; Ali-Shtayeh et al., 2002). In Libya, T. violaceum is responsible for $64 \%$ of cases and $M$. canis is responsible for 25\% (Ellabib et al., 2002). In Saudi Arabia, where the prevalence of tinea capitis is $22 \%$, the principal causative agent is M. canis (Abanmi et al., 2008). In Kuwait, $M$. canis is the predominant dermatophyte isolated in $62.5 \%$ of cases, followed by $T$. violaceum in $19.3 \%$ and $T$. tonsurans in 13.1\% (Razzaq Adel et al., 2007).

Tinea pedis was the second most frequent infection in the hospitalized patients (135 cases). The prevalence of tinea pedis in female gender was twice that of recorded in male gender. Mostly the infection was more common in persons aged between 31-50 years of age. The infection is mainly caused by $T$. mentagrophytes, $T$. rubrum, and unicellular yeast. Several investigators indicated T. rubrum, $T$. mentagrophytes var. interdigitale, and E. floccosum as the common causative agents for tinea pedis (Noble et al., 1998; Stratigos et al., 1999; Crawford et al., 2001; Weinstein and Berman, 2002).

It has been reported that tinea pedis is becoming more common as a result of changes in lifestyle, including in- 


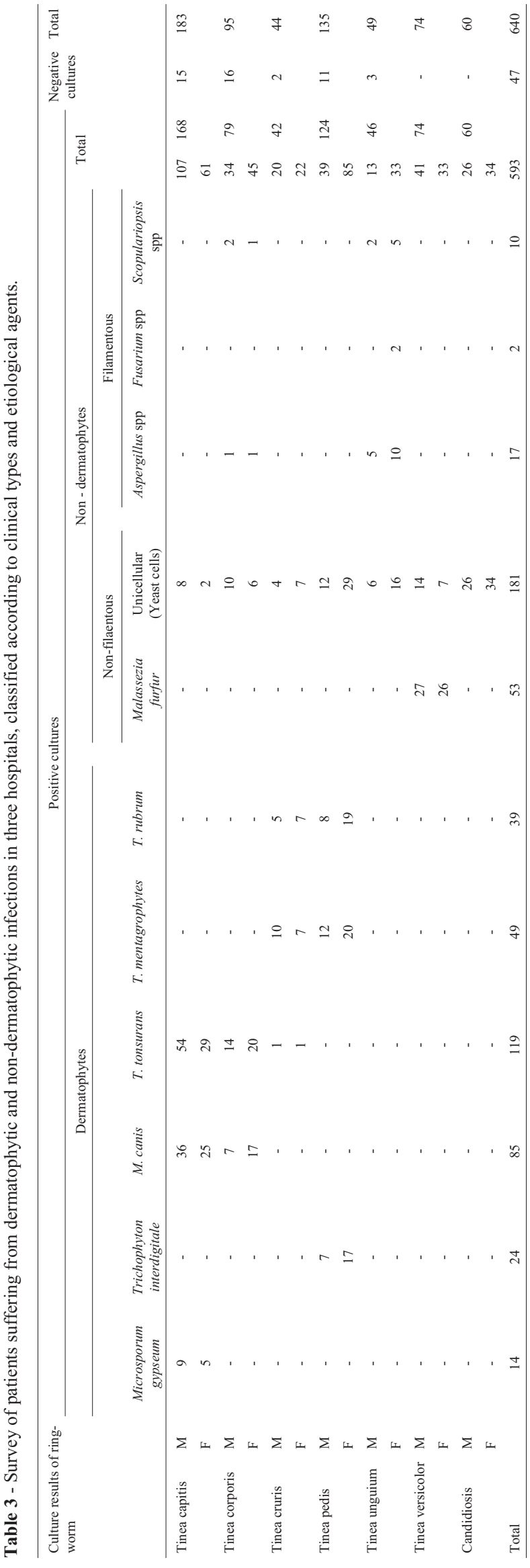

creased urbanization, the use of communal bathing facilities, and occlusive footwear. Moreover, excessive sweating and poor circulation are the most important predisposing factors for tinea pedis (Davis, 1995). The increasing incidence of diabetes and HIV infection are also important contributory factors (Kaur et al., 2008). Certain occupations (miners, soldiers) and recreational activities (marathon runners) place participants at a higher risk of tinea pedis (Gentles and Holmes, 1957; Auger et al., 1993; Djeridane et al., 2007). The high rate of incidence of tinea pedis in female gender may be due to the fact that most of the female patients are villagers and usually walk barefoot without any shoes. Tinea pedis, although not as common in many tropical environments, often shows secondary bacterial infection, and the possibility of mixed fungal and Gram-negative infection of the feet probably because of the climate. This may explain the high cases (41 cases) induced by unicellular yeast. Davis (1995) reported that Candida intertrigo, as an etiological agent of tinea pedis, may occur in patients with conditions that alter host immunity, such as pregnancy, malignancy, diabetes mellitus, and glucocorticoid therapy.

Tinea corporis was the third most common infection among the enrolled patients with a significant incidence in children below 10 years. The site of infection was mostly restricted to face and neck. T. tonsurans and $M$. canis were the main causative agents. Zaki et al. (2009) examined dermatophyte infections in patients referred to the Department of Dermatology, El-Houd El-Marsoud Hospital, Cairo, Egypt during March 2004 to June 2005. Of 506 patients enrolled in their investigation, tinea capitis $(76.4 \%)$, followed by tinea corporis $(22.3 \%)$ and tinea unguium (1.2\%) were the most common infections. The most frequently isolated dermatophyte species was $T$. violaceum, which accounted for most $(71.1 \%)$ of all the recovered dermatophytes, followed by M. canis $(21.09 \%)$, T. rubrum (6.2\%), and M. boullardii (0.49\%). Both E. floccosum and T. tonsurans each were rarely isolated ( $0.24 \%)$. In Tripoli, Libya, Ellabib et al. (2002) reported that tinea corporis accounted for $45.9 \%$ of cases ( $85 \%$ of cases occurred in children below 15 years of age) and T. violaceum was the most common etiological agent, responsible for $44 \%$ (300 cases) of dermatophyte infections.

The present investigation provides data that are valuable for determination of dermatomycoses among the population in Cairo and Giza. This data does an assessment of the prevalence and etiological profiling of the infections. This could help in the estimation of the problem more accurately in future and thence in the prevention of spread of dermatophytosis. Moreover, awareness of the preventive measures regarding public health and maintenance of personal hygiene could reduce the incidence of dermatophy- 
tosis and hence the burden of this disease in the community as a whole.

\section{References}

Abanmi A, Bakheshwain S, El Khizzi N et al. (2008) Characteristics of superficial fungal infections in the Riyadh region of Saudi Arabia. Int J Dermatol 47:229-235.

Al-Duboon AH, Muhsin TM, Al-Rubaiy KK (1999) Tinea capitis in Basrah, Iraq. Mycoses 42: 331-333.

Ali-Shtayeh MS, Salameh AAM, Abu-Ghdeib SI et al. (2002) Prevalence of tinea capitis as well as of asymptomatic carriers in school children in Nablus area (Palestine). Mycoses 45:188-194.

Andrews MD, Burns M (2008) Common Tinea Infections in Children. American Family Physician 77: 1415-1420.

Arenas R, Toussaint S, Isa-Isa R (2006) Kerion and dermatophytic granuloma. Mycological and histopathological findings in 19 children with inflammatory tinea capitis of the scalp. Int J Dermatol 45:215-219.

Arrese JE, Valverde JC, Pierard GE (2005) Revisiting the epidemiology of onychomycoses. Rev Iberoam Micol 22:163166 [in Spanish].

Auger P, Marquis G, Joly J et al. (1993) Epidemiology of tinea pedis in marathon runners: prevalence of occult athlete's foot. Mycoses 36:35-41.

Baran W, Szepietowski JC, Schwartz RA (2004) Tinea barbae. Acta Dermatoven APA 13:91-94.

Baxter M, Rush-Munro FM (1980) The superficial mycoses of man and animals in New Zealand and their diagnosis. Palmerston North, New Zealand (3rd ed). Massey University.

Borman AM, Campbell CK, Fraser M et al. (2007) Analysis ofthe dermatophyte species isolated in the British Isles between 1980 and 2005 and review of worldwide dermatophyte trends over the last three decades. Med Mycol 45:131-141.

Carrillo-Muñoz AJ, Giusiano G, Cardenes D et al. (2008) Terbinafine susceptibility patterns for onychomycosis Causative dermatophytes and Scopulariopsis brevicaulis. Int J Antimicrob Agents 31:540-543.

Chakrabarti A, Sharma SC, Talwar P (1992) Isolation of dermatophytes from clinically normal sites in patients with tinea cruris. Mycopathologia 120:139-141.

Crawford F, Hart R, Bell-Syer S et al. (2001) Athlete's foot and fungally infected toenails. BMJ 322:288-289.

Davis JD (1995) Superficial fungal infections of the skin: Tinea corporis, tinea pedis, and Candida intertrigo. Primary Care Update for OB/GYNS 2:157-161.

Degreef H (2008) Clinical Forms of Dermatophytosis (Ringworm Infection). Mycopathologia 166:257-265.

Denk L (2007) Tinea Corporis. Pediatric Clinical Advisor. $2^{\text {nd }}$ Ed. Mosby Elsevier, Philadelphia, pp. 562-563.

Djeridane A, Djeridane Y, Ammar-Khodja A (2007) A clinicomycological study of fungal foot infections among Algerian military personnel. Clin Exp Dermatol 32:60-63.

Dolenc-Voljc M (2005) Dermatophyte infections in the Ljubljana region, Slovenia, 1995-2002. Mycoses 48:181-186.

Ellabib MS, Agaj M, Khalifa Z et al. (2002) Trichophyton violaceum is the dominant cause of tinea capitis in children in Tripoli, Libya: results of a two year survey. Mycopathologia 153:145-147.
Ellabib MS, Khalifa Z, Kavanagh K (2002) Dermatophytes and other fungi associated with skin mycoses in Tripoli, Libya. Mycoses 45:101-104.

Fernández-Vozmediano JM, Armario-Hita JC (2006) Etiopatogenia y tratamiento de la pitiriasis versicolor. Medicina Clínica 126:7-13.

Gentles JC, Holmes JG (1957) Foot ringworm in coal-miners. Br J Ind Med 14:22-27.

Ghannoum MA, Isham NC (2009) Dermatophytes and dermatophytoses. $2^{\text {nd }}$ ed. Clinical Mycology, pp. 375-384.

Ghannoum M, Isham N, Hajjeh R et al. (2003) Tinea capitis In Cleveland: survey of elementary school students. J Am Acad Dermatol 48:189-193.

Ginter-Hanselmayer G, Weger W, Ilkit M et al. (2007) Epidemiology oftinea capitis in Europe: current state and changing patterns. Mycoses 50:6-13.

Ilkit M, Turac-Bicer A, Ates A et al. (2007) Familial cases of Microsporum canis tinea in Adana, Turkey. J Mycol Med 17:275-278.

Kane J, Summerbell RC (1997) Dermatological mycology: examination of skin, nails, and hair. In: Kane J, Summerbell RC, Sigler L et al. Laboratory handbook of dermatophytes. A clinical guide and laboratory manual of dermatophytes and other filamentous fungi from skin, hair and nails. Star Publishing Company, Belmont, pp. 33-44.

Kaur R, Kashyap B, Bhalla P (2008) Onychomycosis-epidemiology, diagnosis and management. Indian J Med Microbiol 26:108-116.

Kwon-Chung KJ, Bennett JE (1992) Medical mycology. Lea and Febiger, Philadelphia.

Moraes MS, Godoy-Martinez P, Alchorne MM et al. (2006) Incidence of tinea capitis in Sao Paulo, Brazil. Mycopathologia 162:91-95.

Mousavi SAA, Sardoii SS, Shamsadini S (2009) A first case of tinea imbricata from Iran. Jundishapur J Microbiol 2:71-74.

Mugge C, Haustein UF, Nenoff P (2006) Causative agents of Onychomycosis, a retrospective study. J Dtsch Dermatol Ges 4:218-228.

Ngwogu AC, Otokunefor TV (2007) Epidemiology of dermatophytoses in a rural community in Eastern Nigeria and review of literature from Africa. Mycopathologia 164:149158.

Noble SL, Forbes RC, Stamm PL (1998) Diagnosis and management of common tinea infections. Am Fam Physician 58:163-174, 177-178.

Porche DJ (2006) Tinea Pedis: A Common Male Foot Problem. J Nurse Pract 2:152-153.

Raccurt CP, Dorsainvil D, Boncy M et al. (2009) The emergence of Trichophyton tonsurans in Port-au-Prince, Haiti. Med Mycol 47:197-200.

Razzaq Adel AA, Sultan AO, Basmiah AM et al. (2007) Prevalence of tinea capitis in southern Kuwait. Mycoses 50:317320.

Rebell G, Taplin D (1970) Dermatophytes, their recognition and identification. $2^{\text {nd }}$. ed. University of Miami Press, Coral Gables, p 30 .

Rebollo N, López-Barcenas AP, Arenas R (2008) Tinea Capitis. Actas Dermosifiliogr 99:91-100.

Samarai A (2007) Tinea capitis among Iraqi children: public health implication. J Clin Diagn Res 1:476-482. 
Seebacher C, Bouchara JP, Mignon B (2008) Updates on the Epidemiology of Dermatophyte Infections. Mycopathologia 166:335-352.

Simpanya MF (2000) Dermatophytes: Their taxonomy, ecology and pathogenicity. Rev Iberoam Micol, Bilbao, 1-12.

Stratigos A, Stern R, Gonzales E et al. (1999) Prevalence of skin disease in a cohort of shelter-based homeless men. J Am Acad Dermatol 41:197-202.

Summerbell RC (2003) Trichophyton, Microsporum, Epidermophyton, and agents of superficial mycoses. In: Murray PR, Baron EJ, Jorgensen JH et al. (ed.), Manual of clinical microbiology (8th ed.) ASM Press, Washington, D.C. p. 1798-1819.

Svejgaard EL, Nilsson J (2004) Onychomycosis in Denmark: prevalence of fungal nail infection in general practice. Mycoses 47:131-135.
Walsh TJ, Groll AH (1999) Emerging fungal pathogens: evolving challenges to immunocompromised patients for the twentyfirst century. Transpl Infect Dis 1:247-261.

Weinstein A, Berman T (2002) Topical treatment of common superficial tinea infections. Am Fam Physician 65:2095-2102.

Weitzman I, Summerbell RC (1995). The dermatophytes. Clin Microbiol Rev 8:240-259.

Zagnoli A, Chevalier B, Sassolas B (2005) Dermatophyties et dermatophytes. EMC - Pédiatrie 2:96-115.

Zaki SM, Ibrahim N, Aoyama K et al. (2009). Dermatophyte infections in Cairo, Egypt. Mycopathologia 167:133-137.

Associate Editor: Sandro Rogério de Almeida

All the content of the journal, except where otherwise noted, is licensed under a Creative Commons License CC BY-NC. 